Short note

\title{
The influence of the section shape of box-girder decks on the steady aerodynamic yawing moment of double cantilever bridges under construction
}

\author{
S. Pindado*, J. Meseguer, S. Franchini
}

\begin{abstract}
The yawing moment acting on the box-girder deck of reinforced concrete bridges constructed using the balanced cantilever method during the erection stage has been experimentally analyzed by testing different types of bridge cross-sections. Experimental results show that the yawing moment coefficient decreases as the bridge decks become streamlined, and that the yawing moment coefficient reaches a maximum when the bridge deck length is nearly twice the deck width.
\end{abstract}

\section{Introduction}

Over the past few decades, the construction of long-span bridges has increased. Moreover, construction methods have undergone refinement, and have been further developed to cover many special cases. Today, the balanced cantilever method for constructing reinforced concrete box-girder bridges has been recognized as a very efficient way to build bridges without the need for falsework [1]. When designing and 
constructing bridges, one must take into account the different stimuli that can act upon the bridge elements throughout the service life of the structure, including the construction phase. One of these stimuli is the action of wind. This means that the analysis of bridges taking into account the effects of wind during the construction sequence must be performed in order to preserve the safety and serviceability of the bridge. For example, it is recognized that in the case of long-span suspension bridges, aerodynamic stability during erection, particularly during the early stages, is more problematic than in the final configuration [2].

Most efforts devoted to bridge aerodynamics have focused on the aerodynamic stability of suspension and cable-stayed bridges. As a result of such efforts, it can be said that nowadays almost all the necessary tools for the aerodynamic design of long-span bridge decks are available: flutter and buffeting theories have been established and boundary-layer wind tunnels are available to provide the required experimental input data and to validate the outcome of the analyses [3,4].

However, in spite of the above, there are some basic aspects of bridge aerodynamics in which the available data is scarce. This is the case of bridges constructed using the double cantilever method, in which wind forces can cause the deck to rotate during the construction phase. Double cantilever bridges are made during the erection stages of a deck (which is progressively constructed at both ends by sliding concrete forms), and a pier which supports the deck at the middle point (Fig. 1). In this configuration, an aerodynamic yawing moment over the box-girder deck can be induced due to the yaw angle of the incident wind, relative to the bridge deck, or by gusts of wind. Although the erection stage is not very long (from the perspective of classical aerodynamic stability, the design wind speed can hence be reduced to a lower value than that of the final configuration), a rotation of the deck caused by wind can take place due to a lack of continuity in the girder, as the construction condition is often less favorable than in the final state.

As far as we know, there is not much information concerning this problem. Dyrbye and Hansen [5], have proposed a method for calculating the maximum yawing moment produced by the wind on a double cantilever bridge (the method also takes into account the dynamic effects of the wind on the bridge when its yaw angle is zero). Mendes and Branco [6], apply the same method to calculate the wind forces on a bridge over the Douro river (Portugal), including the effect of cantilevers bending. Results concerning the variation of the aerodynamic yawing moment with the length of the deck of a cantilever bridge, and the influence on such a yawing moment on different types of end plates simulating the sliding concrete forms located at both ends of the bridge, have recently been published [7]. That paper concluded that the maximum yawing moment caused by wind action on the box-girder deck tends to grow with the square of the deck length, the sliding concrete forms decreasing the magnitude of the maximum yawing moment.

The influence of both the length and the shape of the box-girder deck during bridge erection using the balanced cantilever method has been experimentally analyzed in a wind tunnel by measuring the mean yawing moment, $M$, caused by the wind flow on four different testing models. Experimental results show that the yawing moment becomes maximum for yaw angles close to $45^{\circ}$, its magnitude 


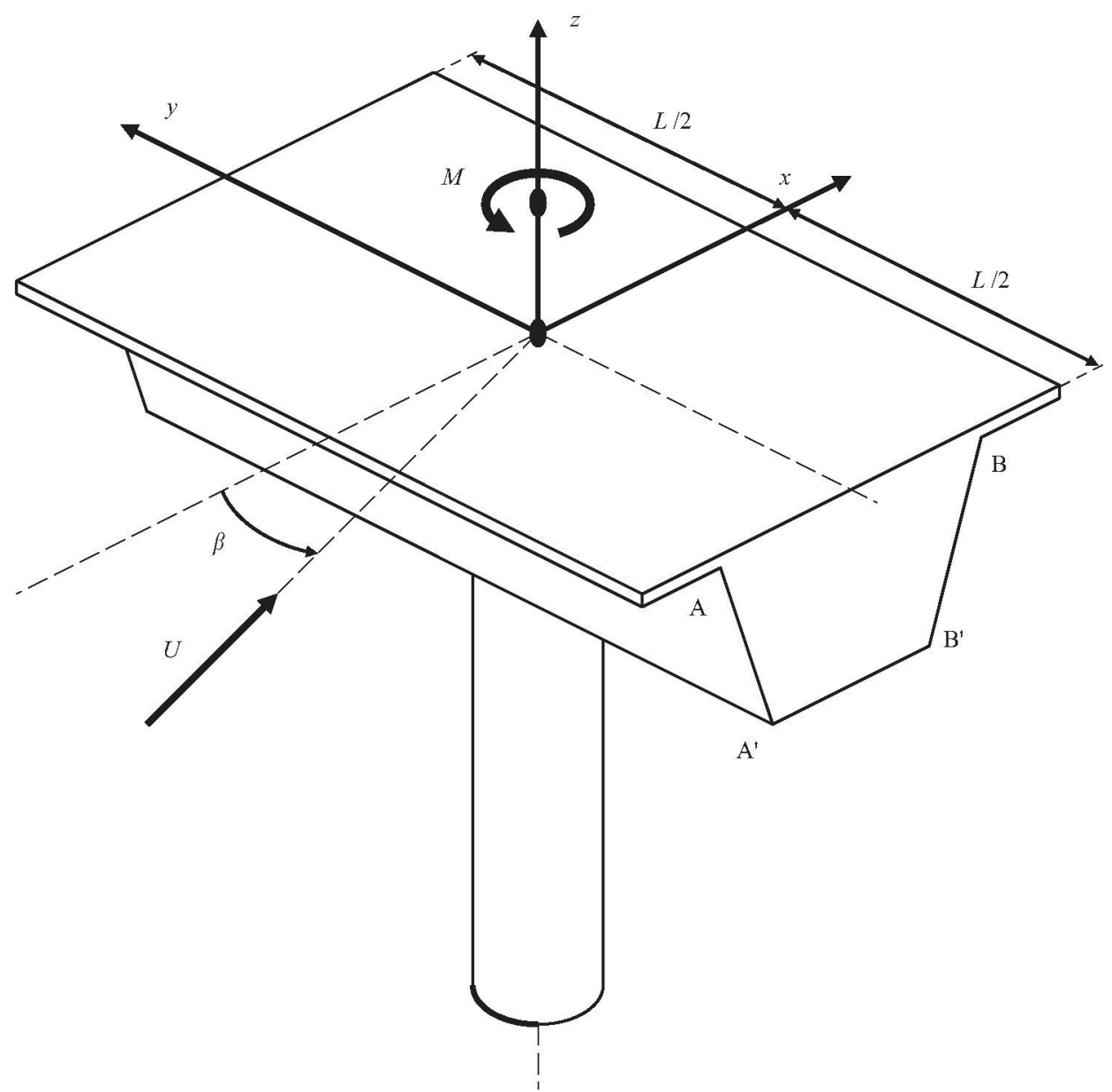

Fig. 1. Double cantilever bridge under a non-zero wind yaw angle, $\beta$. Wind velocity, $U$, and yawing moment over the bridge deck, $M$, are also indicated.

increasing as the shape of the box-girder deck becomes less streamlined (that is, the lateral surfaces of the box-girder deck, $\mathrm{AA}^{\prime}$ and $\mathrm{BB}^{\prime}$ in Fig. 1, become more vertical).

\section{Experimental configuration}

To perform the experiments, four different testing models were built. The crosssections of the different models, namely $\mathrm{B}_{0}, \mathrm{~B}_{1}, \mathrm{~B}_{2}$ and $\mathrm{B}_{3}$, are defined in terms of parameter $a$ in Fig. $2(a=0.02 \mathrm{~m})$. Note that subscript $k$ of the bridge label, $\mathrm{B}_{k}$, also identifies the cross-section geometry from an aerodynamic standpoint, as $k a$ is the horizontal distance between the bottom edge $\left(\mathrm{A}^{\prime}\right.$ or $\left.\mathrm{B}^{\prime}\right)$ and the upper corner ( $\mathrm{A}$ or 

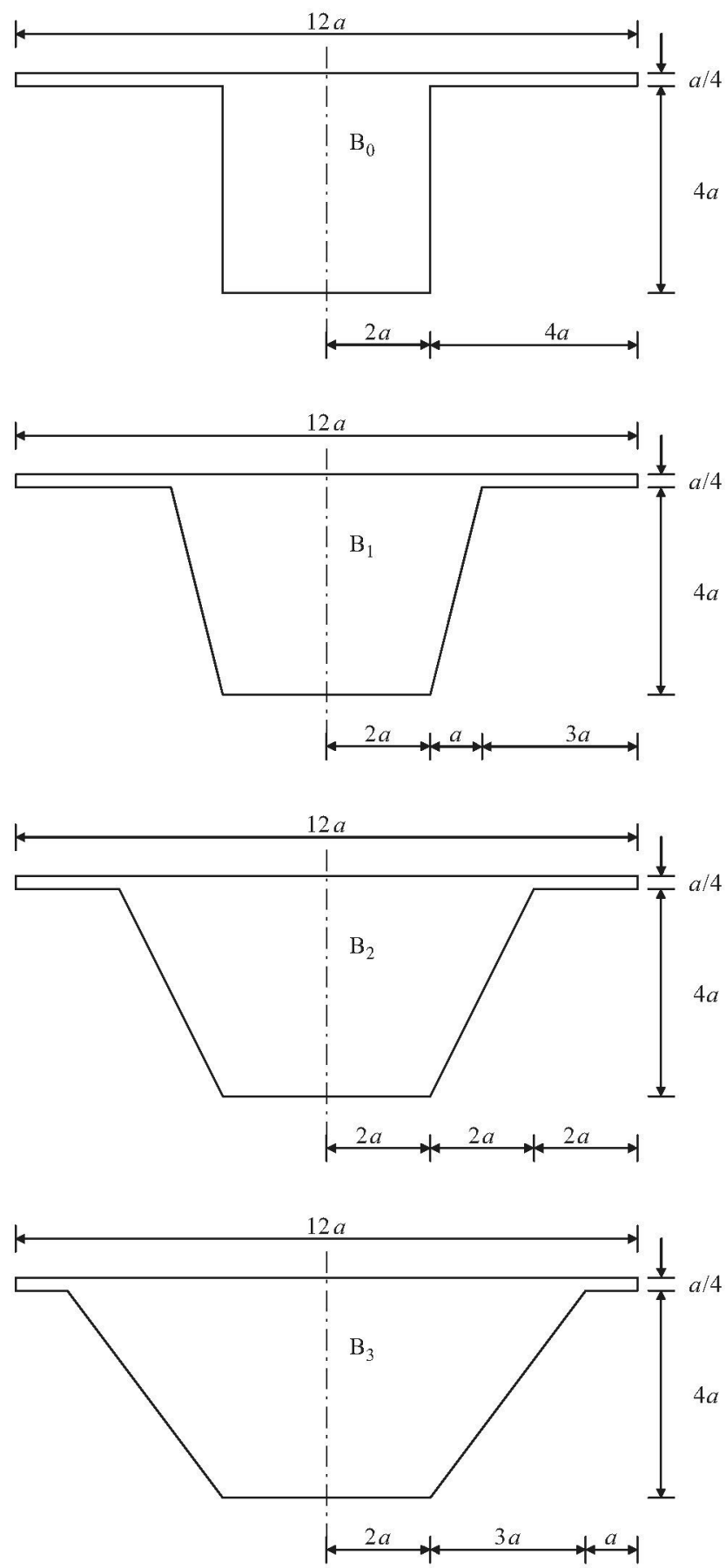

Fig. 2. Sketches of the cross-sections of the different box-girder bridge models used in the wind tests $(a=0,02 \mathrm{~m})$ 
B) of the lateral surfaces of the girder (the cross-section deck becomes more streamlined as shape factor $k$ increases). For each of the testing models, four different box-girder deck lengths have been considered ( $L_{n}=12 n a$, with $n=1,2,3$, 4). During the tests, box-girder decks were supported by a vertical rod, $a / 5$ in diameter and $5 a / 4$ in height, which in turn was anchored to a six-component strain gauge balance.

Measurements were carried out in the A9 wind tunnel at the IDR/UPM, where the test chamber is $1.5 \mathrm{~m}$ in width and $1.8 \mathrm{~m}$ in height. As mentioned earlier, the model was anchored to a Midi Capteurs six-component strain gauge balance, model EX114.45-200, mounted on a NEWPORT RV120-PP-HL rotating platform. This rotating platform made it possible to control the yaw angle of the model with a $\pm 1^{\circ}$ accuracy. The balance and rotating platform were placed inside a tight chamber located under the wind-tunnel floor. This chamber was connected to the wind-tunnel test chamber through a circular hole with a diameter slightly larger than that of the vertical rod, simulating the supporting pier of the bridge deck. Therefore, since the yawing moment caused by the circular rod is virtually negligible (formally this moment must be zero because of the symmetry of the rod with respect to the incident wind), the yawing moment measured by the balance was only generated by the aerodynamic loads acting on the bridge deck. The dynamic pressure inside the test chamber was measured by a calibrated Air Flow 048 Pitot tube connected to a Schaewitz Lucas P3061-2 WG pressure transducer.

It must be pointed out that even in the worst case (a $L_{4}$ length bridge deck at a $0^{\circ}$ yaw angle), the frontal area of the model, including the rod simulating the supporting column, is smaller than $3 \%$ of the wind-tunnel cross-section, so that no provisions for blockage correction of the measured results have been considered. No atmospheric boundary layer simulation was performed, because in this problem the atmospheric boundary layer effects are negligible, provided the bridge is high enough over the ground and the bridge deck is not too thick (so that the wind speed behaves as uniform at bridge height). Therefore, the experiments were performed in a lowturbulence, uniform flow (the turbulence intensity being some $2.5 \%$ ). This lowturbulence testing condition has already been used in similar tests [8], and from the standpoint of measuring averaged wind loads it can even be considered a more severe condition than turbulent flow $[9,10]$. The wind speed was some $20 \mathrm{~m} \mathrm{~s}^{-1}$, which provides a Reynolds number higher than $3 \times 10^{5}$, based on the width of the boxgirder deck, $12 a$.

\section{Experimental results}

The measured yawing moment on the bridge deck, $M$, has been made dimensionless as usual, i.e.:

$$
c_{M}=\frac{M}{\frac{1}{2} \rho U^{2} h L_{n}^{2}},
$$



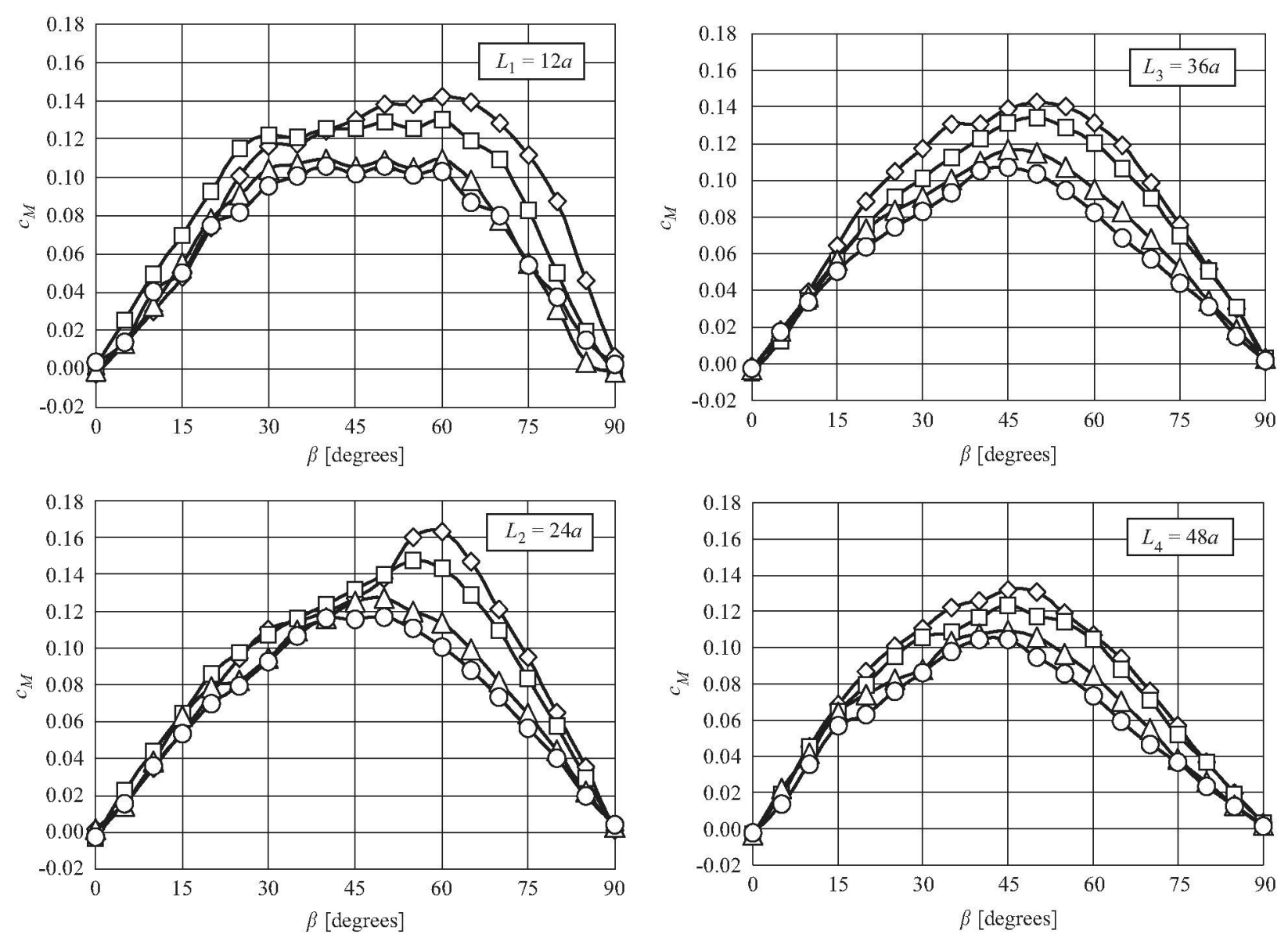

Fig. 3. Yawing moment coefficient, $c_{M}$, versus wind yaw angle, $\beta$, of different box-girder deck models. Labels in the plots identify the deck length, $L_{n}=12 n a$, whereas symbols identify the deck cross-section (as defined in Fig. 2) according to the following key: $B_{0}$ (rhombi), $B_{1}$ (squares), $B_{2}$ (triangles) and $B_{3}$ (circles) 
where $c_{M}$ is the dimensionless yawing moment coefficient, $\rho$ is the air density, $U$ the air flow velocity, $h$ is the height of the box-girder deck ( $h=17 a / 4$, see Fig. 2), and $L_{n}$ its length ( $L_{n}=12 n a$, with $n=1,2,3,4$, as described earlier).

The experimental results are shown in Fig. 3, where the variation of the yawing moment coefficient, $c_{M}$, with the yaw angle, $\beta$, has been represented for different

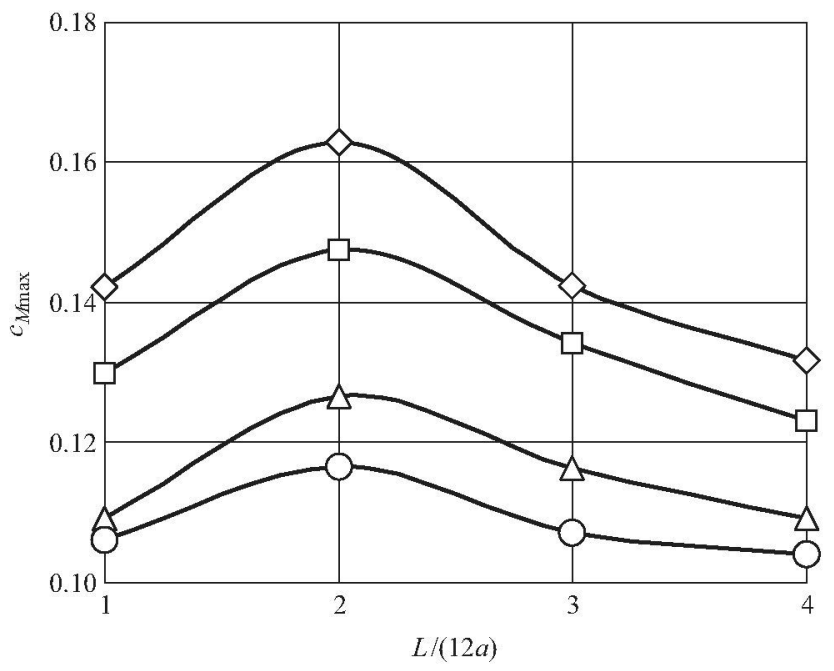

Fig. 4. Maximum yawing moment coefficient, $c_{M \max }$, versus dimensionless deck length, $L /(12 a)$ of different box-girder deck models. Symbols identify the deck cross-section (as defined in Fig. 2) according to the following key: $\mathrm{B}_{0}$ (rhombi), $\mathrm{B}_{1}$ (squares), $\mathrm{B}_{2}$ (triangles) and $\mathrm{B}_{3}$ (circles).

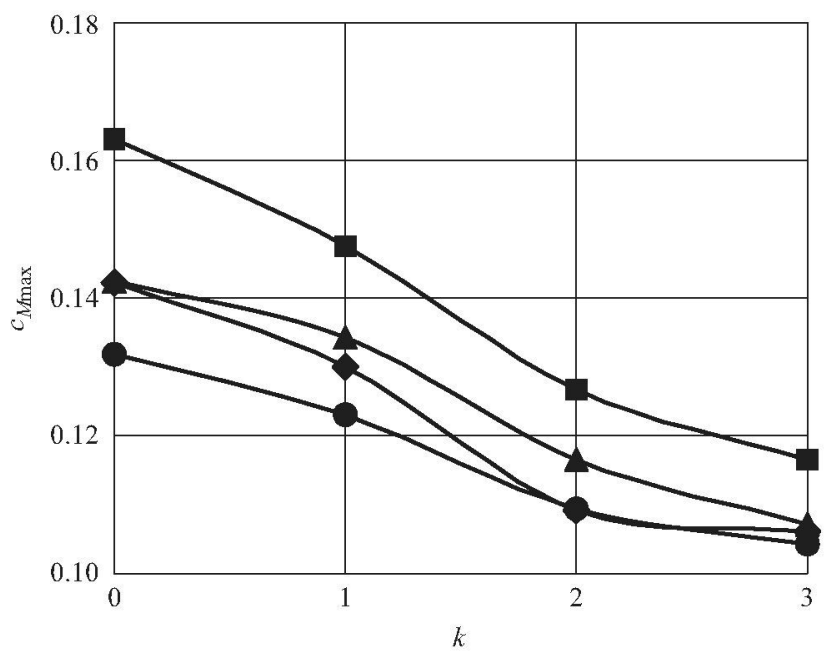

Fig. 5. Maximum yawing moment coefficient, $c_{M \max }$, versus cross-section parameter, $k$, of box-girder deck models $\mathrm{B}_{k}$. Symbols identify the deck length according to the following key: $L_{1}=12 a$ (rhombi), $L_{2}=24 a$ (squares), $L_{3}=36 a$ (triangles) and $L_{4}=48 a$ (circles). 
lengths and cross-sections of the box-girder deck. Obviously, the yawing moment coefficient becomes zero at $\beta=0^{\circ}$ and $\beta=90^{\circ}$, because for these angles of incidence the bridge configuration is symmetrical to the air flow. In general, it can be said that the maximum yawing moment is reached closer to $\beta=45^{\circ}$, as both the deck crosssection becomes more aerodynamically shaped $(k=3)$ and the length of the bridge deck increases. Note that the maximum value moves toward $\beta=60^{\circ}$ as the sharpness of the cross-section increases $(k=0)$ in the case of short bridge decks (this effect is very pronounced for bridges with a length of $L_{2}$ ).
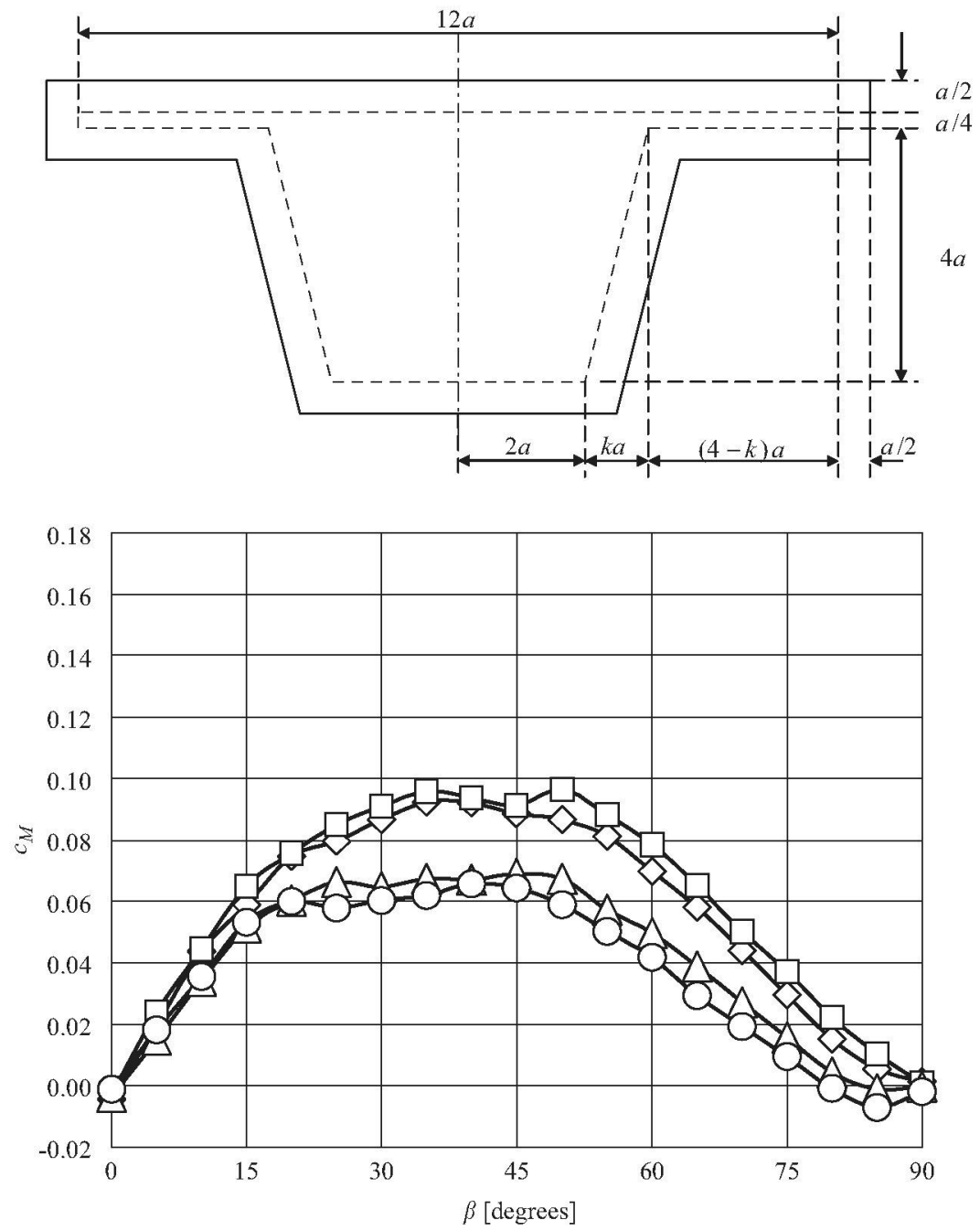

Fig. 6. Yawing moment coefficient, $c_{M}$, versus wind yaw angle, $\beta$, of different box-girder deck models with end plates. The results correspond to a deck length, $L_{4}=48 a$. Symbols identify the deck cross-section (as defined in Fig. 2) according to the following key: $B_{0}$ (rhombi), $B_{1}$ (squares), $B_{2}$ (triangles) and $\mathbf{B}_{3}$ (circles). 
The experimental results are summarized in Fig. 4, which shows the variation of the maximum yawing moment coefficient, $c_{M \max }$, relative to the deck length of the four different types of cross-section decks tested. This maximum yawing moment coefficient is higher for medium-sized decks (for lengths nearly twice the deck width), and the maximum value decreases as shape factor $k$ increases. It must also be pointed out that for a given cross-section of the bridge, the variation of the yawing moment coefficient relative to the bridge length becomes smooth as shape factor $k$ increases. The ratio between the maximum yawing coefficient, $c_{M \max }$, for $L /(12 a)=2$ and $L /(12 a)=4$ is 1.23 for cross-sections where $k=0$, and 1.13 for cross-sections where $k=3$. The dependence of the yawing coefficient on the cross-section shape is clearly stressed in Fig. 5, which shows the maximum yawing moment coefficient, $c_{M \max }$, versus shape factor $k$.

Finally, Fig. 6 shows the results for a bridge with a dimensionless deck length $L /(12 a)=4$, but equipped with end plates to simulate sliding concrete forms. The plates are $a / 4$ thick, and their shape reproduces the deck cross-section, but enlarged to provide a contour in which the sides are $a / 2$ away from the corresponding sides of the deck cross-section. By comparing this plot with the corresponding one in Fig. 3 $(L /(12 a)=4)$, it is clear that end plates reduce the yawing moment coefficient, $c_{M}$. Note that the aerodynamic effect of the end plates becomes dominant for yaw angles close to $\beta=90^{\circ}$, which is explained because end plates generate high suction forces which have opposite sign with respect to those generated by the box-girder deck. (As mentioned earlier, the influence of different types of end plates on the yawing moment coefficient of bridges where the cross-section has a shape factor of $k=1$ has been analyzed elsewhere [7].)

\section{References}

[1] H.-G. Kwak, J.-K. Son, Determination of design moments in bridges constructed by balanced cantilever method, Eng. Struct. 24 (2002) 639-648.

[2] X. Zhang, Investigation on aerodynamic stability of long-span suspension bridges under erection, J. Wind Eng. Ind. Aerodyn. 92 (2004) 1-8.

[3] T. Miyata, Historical view of long-span bridge aerodynamics, J. Wind Eng. Ind. Aerodyn. 91 (2003) $1393-1410$.

[4] F. Ricciardelli, On the wind loading mechanism of long-span bridge deck box sections, J. Wind Eng. Ind. Aerodyn. 91 (2003) 1411-1430.

[5] C. Dyrbye, S.O. Hansen, Wind Effects on Structures, Wiley, New York, 1997.

[6] P.A. Mendes, F.A. Branco, Unbalanced wind buffeting effects on bridges during double cantilever erection stages, Wind Struct. 4 (2001) 45-62.

[7] S. Pindado, J. Meseguer, J.M. Perales, A. Sanz-Andrés, A. Martínez, Wind-induced loads over double cantilever bridges under construction. Proceedings (on CD) of the First International Conference on Bridge Maintenance, Safety and Management, IABMAS 02, Barcelona, 2002, pp. 1-9.

[8] M. Suzuki, K. Tanemoto, T. Maeda, Aerodynamic characteristics of train/vehicles under cross winds, J. Wind Eng. Ind. Aerodyn. 91 (2003) 209-218.

[9] J. Courchesne, A. Laneville, An experimental evaluation of drag coefficient of rectangular cylinders exposed to grid turbulence, J. Fluids Eng. 104 (1982) 523-528.

[10] M.M. Zdravkovich, E. Carelas, Aerodynamics of a covered pedestrian bridge of a trapezoidal section, J. Wind Eng. Ind. Aerodyn. 66 (1997) 141-153. 